\title{
Estudio de visibilidad web 2013 de los museos de Cataluña
}

\author{
Web visibility of the Catalonian museums: the 2013 report
}

Joan-Isidre BADELL (1), Cristòfol RoviRA (2), Miquel TÉRMENS (3)

(1) Doctorando Universidad de Barcelona. Facultad de Biblioteconomía y Documentación. Grupo de Investigación DigiDoc, Universidad Pompeu Fabra, jibadell@gmail.com; (2) Universidad Pompeu Fabra. Departamento de Comunicación. Grupo de Investigación DigiDoc. c/. Roc Boronat, 138, 08018 Barcelona (España), cristofol.rovira@upf.edu; (3) Universidad de Barcelona. Facultad de Biblioteconomía y Documentación. c/. Melcior de Palau, 140, 08014 Barcelona (España), termens@ub.edu

\section{Resumen}

Se presentan los resultados del análisis de visibilidad de las sedes webs de museos de Cataluña realizado en 2013, a partir de los datos obtenidos por el explorador DigiDocSpider en Google. El objetivo global es obtener los datos necesarios para profundizar en la identificación de las buenas prácticas que ayuden a mejorar la visibilidad de cualquier sede web de museo. La metodología utilizada ha sido desarrollada en una doble perspectiva: comparativa y evolutiva con estudios anteriores. Se ha podido comprobar un incremento de visibilidad de los museos catalanes en el período 2006-2013; aunque se ha identificado una pérdida de visibilidad en los últimos cuatro años. En conclusión, los museos más grandes son los que mejor preparados están y mejores resultados obtienen mostrando una distancia muy significativa con los pequeños museos locales.

Palabras clave: Visibilidad. Sitios web. Museos. Cataluña. Enlaces recibidos. PageRank.

\section{Introducción}

En este artículo se presentan los resultados del análisis de visibilidad de las sedes webs de museos de Cataluña realizado en 2013, a partir de los datos obtenidos por el explorador DigiDocSpider en Google. El objetivo global fue obtener los datos necesarios para profundizar en la identificación de las buenas prácticas que pueden ayudar a mejorar la visibilidad de cualquier sede web de museo. Los objetivos específicos del estudio fueron:

- Identificar el cómputo de todas las sedes webs de museos de Cataluña

- Elaborar un ranking de visibilidad de las webs de museos de Cataluña en el año 2013.

- Realizar una comparativa entre los años 2006, 2009 y 2013.

- Identificar las webs que han ganado más visibilidad.

\begin{abstract}
The results of the 2013 analysis of visibility of the museum web sites of Catalonia are presented out of the data obtained by the explorer DigiDocSpider in Google. The main goal is to acquire the necessary data to deepen into the identification of good practices that help improve the visibility of any museum web site. The methodology has focused on a double perspective: comparative and evolutionary with previous research. An increase of visibility of the Catalan museums from 2006 to 2013 has been proved. However, a slight loss of visibility has been confirmed if we compare it to the period 2009-2013. As a conclusion, the biggest museums are better prepared and obtain better results, showing a very significant distance with those small local museums.
\end{abstract}

Keywords: Visibility. Websites. Museums. Catalonia. Backlinks. PageRank.

- Identificar las webs que han perdido más visibilidad.

- Identificar, si es posible, las buenas prácticas llevadas a cabo por los centros.

- Comprobar si existe una relación directa entre el número de enlaces y el PageRank, indicador de Google.

Por otra parte, desde una perspectiva más general, los resultados de este estudio nos permiten avanzar en la valoración del impacto sociocultural de la dimensión digital de los museos de Cataluña y observar si ha habido incrementos o descensos. Creemos importante redundar en la necesidad de abrir espacios de debate y discusión para seguir intentando identificar indicadores fiables y buenas prácticas en visibilidad web.

\section{Actualización bibliográfica}

En la revisión bibliográfica hemos recuperado algunos de los manuales clásicos e imprescindibles de la cibermétria, desde propuestas de 
metodologías de evaluación (Olsina, 1999; Monistrol, 2005; Pardo, 2008; Burnette, 2009); las que aportan métodos de evaluación web más generales (Rodríguez, 2004; Codina, 2000); a las que se centran en algunos aspectos concretos, como la usabilidad (Pallas y Economides, 2008), el diseño centrado en el usuario o en su adecuación para soportar los nuevos dispositivos móviles (Mulholland y otros, 2005; Sharples $y$ otros, 2007).

En la mayoría de trabajos, se incluye el análisis de enlaces recibidos como parámetro de evaluación (Baeza-Yates y Davis, 2004; Baeza-Yates y otros, 2006). En estos últimos años, los trabajos sobre SEO y posicionamiento (Orense y Rojas, 2010; Gupta y Aggawal, 2012) han aumentado considerablemente. Creemos que en la proporción que las empresas y las instituciones dan importancia a la aparición en los primeros puestos de resultados de los buscadores. Es un factor crítico de éxito para cualquier institución, ya sea privada o pública, y la literatura generada así lo refleja. Es indicativo, al respecto, el gran número de blogs y webs personales que tratan el tema de marketing relacionado con las redes sociales principalmente en aspectos económicos, pero también sociales o culturales.

No obstante, no hemos encontrado estudios centrados en el análisis de enlaces de sedes web de museos, exceptuando, aunque desde una perspectiva algo diferente, uno sobre bibliotecas, archivos y museos (Leddy, 2012). En cambio, son abundantes los estudios cibermétricos sobre universidades o instituciones académicas (Aguillo y Orduña, 2013; Ortega y Orduña, 2014). También encontramos estudios sobre semántica web (Stefanidakis y Papadakis, 2011) u otros aspectos, como ciudades turísticas (Rovira y otros, 2010). La ausencia de estudios específicos en el sector museístico y la importancia del parámetro analizado motivaron nuestro interés en la investigación.

\section{Metodología}

Las direcciones web (URL) se han obtenido de los directorios de museos del Ministerio de Educación y Cultura de España y del Departamento de Cultura de la Generalitat de Cataluña. Los datos sobre visibilidad han sido obtenidos mayoritariamente de forma automática utilizando una herramienta de tipo rastreador desarrollada por el Grupo de Investigación DigiDoc de la Universidad Pompeu Fabra, conocido como DigiDocSpider, citado anteriormente. El programa ha sido configurado para extraer datos de forma no intrusiva y abusiva de los buscadores.
La visibilidad es el número de enlaces que recibe un sitio web y ofrece una idea del impacto del sitio web en Internet (Bray, 1996; Aguillo, 2000; Alonso Berrocal, 2004). Los enlaces que recibe un sitio web se conocen como enlaces recibidos, entrantes o backlinks en inglés.

En algunos casos, para calcular la visibilidad se contabilizan los enlaces entrantes que apuntan a todas las páginas del sitio web, pero en otras ocasiones solo se consideran los enlaces que apuntan a la página de inicio. Por otra parte, a veces se consideran todos los enlaces entrantes y en otras ocasiones solamente los que provienen de páginas externas y por tanto se eliminan los autos enlaces.

La visibilidad está fuertemente relacionada con otro indicador cibermétrico de gran importancia, el factor de impacto (Rodríguez i Gairín, 1997; Ingwersen, 1998). El factor de impacto web intenta reproducir en la web el clásico factor de impacto de una publicación científica y se calcula dividiendo el número de enlaces entrantes que apuntan a alguna página de la web por el número total de páginas del sitio web. Algunos autores, también en el factor de impacto, eliminan los auto-enlaces; no obstante, se trata de un indicador poco usado que ha recibido fuertes críticas (Noruzi, 2006).

En nuestro estudio se ha utilizado el indicador de visibilidad, considerando los enlaces recibidos que apuntan a la página de inicio en lengua catalana del web analizado. El catalán es la lengua utilizada como principal en todos los museos catalanes. Siendo las versiones en castellano e inglés, en muchos casos, aunque no en todos, reducidas, sobre todo la versión en lengua inglesa.

Para la elaboración del estudio se han recogido los datos sobre la visibilidad de los sitios web de museos durante los meses de noviembre y diciembre de 2012. Una posterior revisión manual realizada en abril de 2013 fija la fecha final de los resultados obtenidos.

Los datos se han analizado desde una doble perspectiva: comparativa y evolutiva. Se han estudiado los datos de los sitios web de los museos de Cataluña obtenidos en 2006, 2009 para la realización de las tablas comparativas y para analizar su evolución.

Por último, es necesario hacer una aclaración previa respecto a los artículos publicados anteriormente por el Grupo de Investigación DigiDoc (1) (Digital Documentation) de la Universidad Pompeu Fabra. En concreto, se publicaron sendos artículos centrados en visibilidad de sedes web de museos (Monistrol, Codina y Rovira, 
2006; Badell y Rovira, 2010), obteniendo los datos a través del programa rastreador DigiDocSpider (Rovira, 2007) en Yahoo. Para ello, se ejecutó una búsqueda directa en el formulario de Yahoo Site Explorer con los siguientes parámetros: "enlaces entrantes", "excepto de ese dominio" y "solo en URL". Sin embargo, Yahoo ha cerrado su buscador recientemente. DigiDocSpider recopiló al mismo tiempo que en Yahoo los datos en Google de forma automática utilizando más de cien indicadores relativos a aspectos básicos de los sitios web: accesibilidad, metadatos, posicionamiento, visibilidad y calidad del código XHTML. Esta recopilación ha sido la utilizada para este artículo. Por lo tanto, no sería adecuada una comparativa ni una relación con los artículos publicados anteriormente, puesto que los datos y metodología son diferentes. En su momento, creímos que Google ofrecía datos menos fiables que Yahoo. Consecuentemente no hemos realizado una actualización de los trabajos anteriores, sino que hemos desarrollado una investigación nueva, con metodología nueva y hemos obtenido datos y resultados completamente nuevos y diferentes.

\section{Resultados}

La tabla I, ordenada de mayor a menor según la cantidad de enlaces recibidos, muestra el resultado obtenido en 2013. En primer lugar, se evidencia una correspondencia entre la cantidad de enlaces recibidos con el tamaño e importancia del museo. Ocupan los primeros puestos los grandes centros, sin excepción. En segundo lugar, los cero o muy pocos enlaces de algunos museos pueden explicarse, en algunos casos, por ser centros pequeños que no muestran interés o están faltos de recursos humanos, y en otros casos, como el de los dos museos de Historia de Barcelona (Monestir de Pedralbes y Parc Güell), por el cambio reciente de denominación y url. También hemos observado museos con enlaces rotos, hecho que acaba de explicar su baja cantidad de enlaces de entrada.

\begin{tabular}{lr}
\hline Museo (ordenación por cantidad de enlaces) & Enlaces recibidos \\
\hline Fundació Miró & 401 \\
\hline Fundació Tàpies & 295 \\
\hline Museu Nacional d'Art de Catalunya & 294 \\
\hline Museu Picasso & 284 \\
\hline Museu d'Art Contemporani de Barcelona & 278 \\
\hline Fundació Gala- Salvador Dalí & 218 \\
\hline Museu Nacional de la Ciència i la Tècnica de & 211 \\
Catalunya & 188 \\
\hline Museu d'Història de Barcelona & 171 \\
\hline Museu de Ciències Naturals de Barcelona & 154 \\
\hline Museu Marítim de Barcelona & 131 \\
\hline Museu d'Història de Catalunya & 130 \\
\hline Museu d'Arqueologia de Catalunya &
\end{tabular}

\begin{tabular}{|c|c|}
\hline Museu d'Art Modern de Tarragona & 82 \\
\hline Museu Episcopal de Vic & 68 \\
\hline Museu de la Música & 59 \\
\hline Museu del Ferrocarril de Vilanova i la Geltrú & 50 \\
\hline Museu Frederic Marès & 46 \\
\hline Museu del Joguet de Catalunya (Figueres) & 45 \\
\hline Museu de la Pesca de Palamós & 43 \\
\hline Museu Darder de Banyoles & 40 \\
\hline $\begin{array}{l}\text { Biblioteca Museu Víctor Balaguer (Vilanova i } \\
\text { la Geltrú) }\end{array}$ & 37 \\
\hline IMGPCN - Museu de Gavà & 35 \\
\hline Museu del Cinema (Girona) & 35 \\
\hline Museu de Badalona & 34 \\
\hline Museu de l'Empordà & 34 \\
\hline Museu Comarcal de l'Urgell & 33 \\
\hline Museu d'Història de Girona & 31 \\
\hline Museu d'Art Jaume Morera (Lleida) & 27 \\
\hline Museu Comarcal de Cervera & 19 \\
\hline Museu Comarcal de Manresa & 18 \\
\hline Museu Tèxtil i d'Indumentària & 16 \\
\hline Museu de Montserrat & 15 \\
\hline Museu Municipal Josep Aragay (Breda) & 15 \\
\hline Museu Pau Casals & 15 \\
\hline Museu del Suro de Palafrugell & 14 \\
\hline Museu de Cardedeu & 13 \\
\hline Museu Deu (Vendrell) & 13 \\
\hline Museu Etnològic del Montseny, la Garbella & 13 \\
\hline Museu Etnogràfic de Ripoll & 9 \\
\hline Museu del Futbol Club Barcelona & 8 \\
\hline Museu Diocesà de la Seu d'Urgell & 6 \\
\hline Museu Geològic del Seminari de Barcelona & 6 \\
\hline Museu d'Història de Sant Feliu de Guíxols & 5 \\
\hline Museu d'Art de Girona & 4 \\
\hline Museu de la Noguera & 4 \\
\hline Museu de Mataró & 4 \\
\hline Museu Comarcal de la Conca de Barberà & 3 \\
\hline Museu Molins de la Vila (Conca de Barberà) & 3 \\
\hline $\begin{array}{l}\text { Museu de la Pell d'Igualada i Comarcal de } \\
\text { l'Anoia }\end{array}$ & 2 \\
\hline Museu d'Història de Cambrils & 2 \\
\hline Museu Municipal de Tossa de Mar & 2 \\
\hline Museu Romàntic Can Papiol & 2 \\
\hline Museu Arqueològic Comarcal de Banyoles & 1 \\
\hline Museu d'Arqueologia Salvador Vilaseca & 1 \\
\hline Museu del Vidre de Vimbodí & 1 \\
\hline Museu Diocesà de Tarragona & 1 \\
\hline Museu Municipal Vicenç Ros (Martorell) & 1 \\
\hline Catedral de Girona & 0 \\
\hline L'Enrajolada Casa-Museu Santacana & 0 \\
\hline Museu Agrícola de Cambrils & 0 \\
\hline $\begin{array}{l}\text { Museu d'Història de Barcelona Monestir de } \\
\text { Pedralbes }\end{array}$ & 0 \\
\hline Museu d'Història de Barcelona Parc Güell & 0 \\
\hline Museu de l'Institut Botànic de Barcelona & 0 \\
\hline Museu de Terrassa & 0 \\
\hline Museu d'Història de Sabadell & 0 \\
\hline Museu Municipal de Nàutica del Masnou & 0 \\
\hline Museu Torre Balldovina & 0 \\
\hline $\begin{array}{l}\text { Museu-Arxiu Municipal de Calella Josep M. } \\
\text { Codina i Bagué }\end{array}$ & 0 \\
\hline
\end{tabular}

Tabla I. Ranking de los museos de Cataluña analizados (2013) 
En la tabla II hemos agrupado, en forma de comparativa, los doce centros con mejores resultados en 2013. Todos superan el centenar de enlaces, respecto a los obtenidos en 2006 (Monistrol y otros), en una perspectiva de siete años. Los resultados se muestran en valores absolutos.

Destaca la pérdida importante de enlaces, difícil de explicar, en la Fundació Miró, el Museu d'Art Contemporani de Barcelona y la Fundació GalaDalí. En cambio, el Museo Marítimo de Barcelona ha triplicado sus resultados. También aumentan el Museo Picasso, el Museu de Ciències Naturals de Barcelona (en 2006 con el nombre de Museo de Zoología), el de Història de Barce-
Iona y el de Història de Catalunya. Son muy significativos los aumentos de 0 en 2006 a los actuales 295, de la Fundació Tàpies y los del Museu Nacional de la Ciència i la Tècnica de Catalunya o los del Museu d'Arqueologia. Otros centros menores tienen incrementos considerables, no incluidos en la tabla II, como el Museu d'Història de Girona que ha pasado de 4 enlaces recibidos en 2006 a los 31 del estudio de 2013. Así mismo la Biblioteca Museo Víctor Balaguer ha aumentado de 7 hasta los actuales 37. En global, el incremento es notorio y creemos que en la mayoría de los casos obedece a una política estratégica premeditada para aumentar la visibilidad y la presencia de los centros en Internet.

\begin{tabular}{lrrrrr}
\hline Museo & 2006 & 2013 & Diferencia & Dif. acumulada & \% acumulado \\
\hline Fundació Miró & 628 & 401 & -227 & -227 & $-18,12$ \\
\hline Fundació Tàpies & 0 & 295 & 295 & 68 & 5,43 \\
\hline Museu Nacional d'Art de Catalunya & 206 & 294 & 88 & 156 & 12,45 \\
\hline Museu Picasso & 191 & 284 & 93 & 249 & 19,87 \\
\hline Museu d'Art Contemporani de Barcelona & 443 & 278 & -165 & -168 & 6,70 \\
\hline Fundació Gala - Salvador Dalí & 386 & 218 & -168 & -84 & $-6,70$ \\
\hline Museu Nacional de la Ciència i la Tècnica de Catalunya & 0 & 211 & 211 & 127 & 10,14 \\
\hline Museu d'Història de Barcelona & 13 & 188 & 175 & 302 & 24,10 \\
\hline Museu de Ciències Naturals de Barcelona & 35 & 171 & 136 & 438 & 34,96 \\
\hline Museu Marítim de Barcelona & 48 & 154 & 106 & 544 & 43,42 \\
\hline Museu d'Història de Catalunya & 40 & 131 & 91 & 635 & 50,68 \\
\hline Museu d'Arqueologia de Catalunya & 0 & 130 & 130 & & 765 \\
\hline Total de los 12 mejores museos & 1.990 & 2.755 & 765 & & 61,05 \\
\hline Total global de los 66 museos estudiados & 2.376 & 3.629 & 1.253 & &
\end{tabular}

Tabla II. Comparativa de estudios de visibilidad 2006-2013 de los 12 mejores

Las medias globales de los análisis realizados, nos muestran un aumento en el período 20062013. En estos años los museos catalanes han aumentado de promedio el número de enlaces en 1.253 , lo que supone un aumento del $112,3 \%$. Sin embargo, si analizamos el período 2009-2013 (Tabla III), los resultados globales muestran una pérdida de enlaces de 1.153, un $24 \%$. Es decir, en la perspectiva de siete años se mantiene un incremento, que se dio básicamente entre 2006 y 2009. En cambio, durante este último período ha habido una pérdida significativa. Creemos que no se trata de una bajada de interés ni de pérdida de buenas prácticas por parte de los centros, sino que es resultado de la reducción de recursos que ha llevado a la cancelación de proyectos, actividades y reducciones de personal. Otras circunstancias a tener en consideración es la existencia de enlaces rotos, cambios de nombre y de url, así como algún centro cerrado por reformas.
Otro dato destacable es la concentración que se produce: Los 12 museos más importantes concentran casi el $76 \%$ del total de enlaces. No obstante, esta cifra ha bajado respecto a la de 2006, cuando la concentración era del $84 \%$. ¿Podemos pensar que los museos pequeños están trabajando en la mejora de su visibilidad? Algunos ejemplos encontrados van en esta dirección, aunque habrá que comprobarlo en el futuro.

\begin{tabular}{cccc}
\hline Total & 2009 & 2013 & \% acumulado \\
\hline-1.138 & 4.767 & 3.629 & $-24 \%$ \\
\hline
\end{tabular}

Tabla III. Comparativa de estudios de visibilidad 2009-2013 global

Podemos ver (Tabla IV), que casi todos los museos que han ganado más visibilidad también han aumentado su PageRank. Ninguno de los centros ha perdido ranking de PageRank. La Fundació Tàpies sigue con cero - entendemos 
que su página web veta los rastreadores- y el Museu d'Art Modern de Tarragona mantiene su indicador en 5 . Los resultados han vuelto a confirmar la idea básica del posicionamiento web: el PageRank depende en buena parte, pero no exclusivamente, del número de enlaces recibidos. De esta manera puede explicarse en parte el caso de la Fundación Miró, que incrementa su indicador de 5 a 6 pese a perder enlaces significativamente. Los resultados son dispares: algunos centros se mantienen, aunque la mayoría pierden ranking en PageRank al mismo tiempo que pierden enlaces recibidos.

\begin{tabular}{lccc}
\hline Museos que han ganado visibilidad y PageRank & 2013 & 2009 & 2006 \\
\hline Fundació Tàpies & 0 & 0 & 0 \\
\hline $\begin{array}{l}\text { Museu Nacional de la Ciència i la Tècnica de } \\
\text { Catalunya }\end{array}$ & 6 & 6 & 4 \\
\hline Museu d'Història de Barcelona & 6 & 5 & 4 \\
\hline Museu de Ciències Naturals de Barcelona & 6 & 1 & 4 \\
\hline Museu d'Arqueologia de Catalunya & 6 & 5 & 2 \\
\hline Museu Marítim de Barcelona & 5 & 0 & 0 \\
\hline Museu Picasso & 6 & 6 & 6 \\
\hline Museu d'Història de Catalunya & 6 & 6 & 5 \\
\hline Museu Nacional d'Art de Catalunya & 6 & 6 & 6 \\
\hline Museu d'Art Modern de Tarragona & 5 & 5 & 5 \\
\hline Museos que han perdido visibilidad y PageRank & 2013 & 2009 & 2006 \\
\hline Fundació Miró & 6 & 5 & 6 \\
\hline Fundació Gala- Salvador Dalí & 6 & 6 & 6 \\
\hline Museu d'Art Contemporani de Barcelona & 6 & 7 & 6 \\
\hline Museu del Futbol Club Barcelona & 4 & 4 & 4 \\
\hline Museu Comarcal de la Conca de Barberà & 0 & 4 & 4 \\
\hline Museu Tèxtil i d'Indumentària & 4 & 5 & 5 \\
\hline Museu-Arxiu Municipal de Calella Josep M. & 3 & 2 & 4 \\
Codina i Bagué & & & \\
\hline Museu de la Pell d'Igualada i Comarcal de & 2 & 0 & 4 \\
l'Anoia & 2 & 4 & 3 \\
\hline Museu d'Arqueologia Salvador Vilaseca & 5 & 5 & 5 \\
\hline Museu del Joguet de Catalunya (Figueres) & & & \\
\hline
\end{tabular}

Tabla IV. PageRank de los museos con mayor aumento y pérdida de visibilidad

\section{Conclusiones}

Creemos que hemos logrado alcanzar los objetivos marcados al haber logrado identificar el cómputo de todas las sedes webs de museos de Catalunya; hemos elaborado los rankings de visibilidad e identificado las webs que han ganada y las que han perdido visibilidad tal y cómo habíamos previsto; y hemos podido comprobar que es en los grandes museos - dónde trabajan especialistas web, y por lo tanto, disponen de políticas más o menos planificadas de SEOdónde se concentra el mayor número de enlaces $(76 \%)$.

En conclusión, los museos catalanes aumentaron visibilidad en el período 2006-2013 en un $112,3 \%$. No obstante, el período 2009-2013 registra una pérdida de visibilidad del $24 \%$, lo que nos lleva a poner un toque de atención y alerta a las estrategias de posicionamiento y SEO llevadas a cabo recientemente por los museos. Está pérdida coincide con el período de crisis y recortes presupuestarios. Esta podría ser una de las causas más plausibles que explicaran el retroceso, aunque no la única, cómo apuntábamos anteriormente.

En cuanto al PageRank, los datos obtenidos no permiten una afirmación categórica, aunque, en nuestra opinión, existe una cierta correlación entre los enlaces de entrada y el indicador de Google, en concordancia sobre lo que se conoce sobre su diseño. En cualquier caso, constituye una interesante línea de investigación para el futuro. No es descartable que los valores sobre el número de resultados que ofrecen los buscadores contengan errores. Son estimaciones aproximadas de los resultados reales y no cálculos precisos de un conjunto de registros de una base de datos. Los buscadores potencian la rápida respuesta a costa de no recuperar físicamente todos los resultados pertinentes; y, por tanto, se ven en la obligación de aplicar un algoritmo de estimación del número total de resultados. No sabemos con qué margen de error nos estamos moviendo, tampoco si se han producido cambios en los últimos años en los algoritmos de estimación del buscador que puedan haber afectado a esta investigación.

Pensamos que el escenario más plausible es aquél en el que el algoritmo de estimación no se haya modificado de forma significativa $y$, por tanto, el margen de error de la recogida de datos de los años 2006 y 2009 sea equiparable al posible error del 2012-2013.

Creemos que quedan abiertas nuevas líneas de investigación, en especial para identificar si hay diferencias significativas en función del tipo, el tamaño o el presupuesto de las instituciones museísticas, sobre todo en tiempos de crisis. Estos nuevos estudios deben profundizar en la identificación de buenas prácticas para mejorar la visibilidad y en la comprobación de los valores e indicadores, así como en la obtención de datos de los buscadores -en nuestro caso, de Google mientras mantenemos Yahoo u otros sistemas en observación.

\section{Notas}

(1) Grupo de Investigación DigiDoc, Universidad Pompeu Fabra, Departamento de Comunicación, .http://www.upf. edu/digidoc/presentaciodigidoc/ 


\section{Referencias}

Aguillo, I. F. (2000). Proposal for a research agenda for cibermetric studies. // 7th International Conference of the International Society for Scientometrics and Informetrics, Coloma (México).

Aguillo, I. F.; Orduña-Malea, E. (2013). The Ranking Web and the "World-Class" Universities. // Building WorldClass Universities. 197-217.

Alonso Berrocal, J. L.; et al. (2004). Cibermetría: nuevas técnicas de estudio aplicables al web. Gijón: Trea.

Baeza-Yates, R.; Davis, E. (2004). Web page ranking using link attributes. // Proceedings International Web Wide World Conference.

Baeza-Yates, R.; Boldi, P.; Castillo, C. (2006). Generalizing PageRank: damping functions for link-based ranking algorithms. // Proceedings Annual ACM Conference on Research and Development in Information Retrieval. 308-315.

Badell, J.-I.; Rovira, C. (2010). Visibilidad de las sedes web de los museos de Cataluña. // Revista Española de Documentación Científica. 33:4, 531-552.

Burnette, A. (2009). Redesigning your museum's web site: a survivors' guide. // Trant, J.; Bearman, D. (editores). Museums and the Web 2009: proceedings. Archives \& Museum Informatics.

Codina, L. (2000). Evaluación de recursos digitales en línea: conceptos, indicadores y métodos. // Revista española de documentación científica. 23:1, 9-44

Directorio de los Museos de España. Madrid: Ministerio de Cultura. http://www.mcu.es/directoriomuseo/loadSearch do?cache=init\&layout=directoriomuseo\&language=es $\quad(2$ de noviembre de 2012).

Gupta, S.; Aggarwal, A. (2012). Estudio del posicionamiento optimizado web.

Ingwersen, P. (1998). The calculation of web impact factors. // Journal of Documentation. 54:2, 236-243.

Leddy, Chuck. (2012). Linking libraries, museums, archives. // Harvard gazette. 2-3.

Monistrol, R. (2005). Difusió cultural i webs de museus on line: estat de la qüestió. Doctorado bienio 2001-2003 en Comunicación Social, UniversitatPompeu Fabra. Trabajo de investigación.

Monistrol, R.; Rovira, C.; Codina, L. (2006). Sitios Web de museos de Cataluña: análisis y propuesta de evaluación. // Hipertext.net. 4.

Mulholland, P.; Collins, T.; Zdrahal, Z. (2005). Bletchley park text: using mobile and semantic web technologies to support the post-visit use of online museum resources. // Journal of Interactive Media in Education. 24, 1-21.

Noruzi, A. (2006). The web impact factor: a critical review. // The Electronic Library. 24:4, 490-500.

Olsina, L. A. (1999). Metodología cuantitativa para la evaluación y comparación de la calidad de sitios web. // Facultad de Ciencias Exactas, Universidad de la Plata (Argentina). Tesis doctoral.

Ortega J. L.; Orduña-Malea, E.; Aguillo I. F. Are web mentions accurate substitutes for inlinks for Spanish universities? // Online Inf Rev. 38:1, 59-77.

Orense, M.; Rojas, O. (2010) SEO cómo triunfar en Buscadores. Madrid: ESIC

Pallas, J.; Economides, A. (2008). A. Evaluation of art museums' web sites worldwide. // IOS Press. 28:1.

Pardo Kuklinski, H. (2008). Sitios web institucionales de museos: un modelo de análisis hacia la web 2.0. // Mateos Rusillo, S.M. (coord.). La comunicación global del patrimonio cultural. Gijón: Trea. 380-396.

Patrimoni. Gencat. Museus. Barcelona: Generalitat de Cataluña http://cultura.gencat.net/museus/muscerca1.asp (2 de noviembre de 2012).

Sharples, M. y otros (2007). An evaluation of my art space: a mobile learning service for school museum trips. // Proceedings of my Learn, Melbourne, Australia, 2007.

Rodríguez, Á. F. Z.; Paniagua, C. G. F.; Berrocal, J. L. A. (2004). Cibermetría: nuevas técnicas de estudio aplicables al Web. // Rev. Esp. Doc. Cient. 27:3.

Rodríguez i Gairín, J. M. Valorando el impacto de la información en internet. http://www.kronosdoc.com/jm.htm.

Rovira, C. (2007). Artículo M529. Rastreo automático: DigiDocSpider. // Rovira, C.; Codina, L. (directores.). Documentación digital. Barcelona: Área de Ciencias de la Documentación. Departamento de Periodismo y de Comunicación Audiovisual. Universidad Pompeu Fabra, http://www.documentaciondigital.org.

Rovira, C.; et al. (2010). Search Engine Positioning of Spanish Official Websites of Provincial Capitals on Searches of City Brand. // El Profesional de la Informacion 19:3, 277-284.

Stefanidakis, M.; Papadakis, I. (2011). Linking the (un)linked data through backlinks. // Proceedings of the International Conference on Web Intelligence Mining and Semantics WIMS. 11:1.

Enviado: 2014-04-21. Segunda versión: 2014-07-10 Aceptado: 2014-07-10 